\title{
Electricity Generation Using Spring-Powered Floor Pad
}

\author{
Engr. Lorinda E. Pascual ${ }^{1}$, Engr. Christopher M. Ladignon ${ }^{2}$, Engr. Noel T. Florencondia ${ }^{3}$, Engr. Erbert Alison \\ J. Benedicto ${ }^{4}$, Engr. Francis Victor B. Bote ${ }^{5}$, Engr. Jerome M. Yabot ${ }^{6}$ \\ 1,2,3,4,5,6 Nueva Ecija University of Science and Technology \\ Gen. Tinio Street Cabanatuan City 3100
}

Corresponding Author: Engr. Lorinda E. Pascual

\begin{abstract}
Walking is the most common activity in our daily life. When we walk, we lose energy to the floor surface. Vibration is one form of energy that is transferred from our weight on to the floor surface during every step. This energy can be harvested and converted into electrical energy. This research addressed the design and construction of a power generating floor pad which can be used to harvest electricity from human footsteps. The electric generating floor pad features springs mounted on its four corners. When somebody walks though the surface of the floor pad, the springs will be compressed because of the weight of the person causing it to dip down slightly. The shaft of the permanent magnet generator will rotate then rotate, thus voltage is generated. The generator can be connected to a battery so as to store electrical energy. Test performed on the device indicates that it is capable of converting human footsteps to a useful electrical energy to power small electrical devices. The magnitude of the generated voltage can be maximized by applying more force on the floor pad. The discharging time of the battery is longer when there are more footsteps applied to the floor pad. The device can be conveniently installed in the doorways of buildings or other heavy traffic areas. Through this research project, a new option for harnessing green electricity by footsteps is made available focusing on the use of springs and permanent magnet generators.
\end{abstract}

Keywords: voltage, electrical energy, generator, electricity

\section{INTRODUCTION}

Electricity has been an essential commodity in our modern day living including economic activities for nation development. According to Ripple (2000), household consumption of commercial energy is widely viewed as enhancing standard of living and that there is a close relationship between the energy used per person and his standard of living. In this generation of an information technology age, there are numerous inventions which are electrical in nature making everyday living more convenient. The society is so much dependent upon the use of electricity that it has become part and parcel of our life.

As a result of population growth and economic development, the need for electricity has been increasing while its source has been becoming less reliable due to greater awareness of the threats posed by climate change. In no time, the supply and generation of energy are on the verge of shortage with growing energy consumption and dwindling energy resources. If no proper actions are taken today, the energy supply will not meet the electricity requirements.

Currently, our country is facing concerns over resource inadequacy in its power sector, as the nation is challenged to add supply quickly enough to keep up with growing demand. In line with the issues of power shortage, one of the strategic solutions in ensuring stable and quality supply of energy is the utilization of an alternative source of energy.

The Philippines, according to the 2010 United Nations Population Fund report, ranked as 12th most populous country in the world with an estimated population of more than 100 million people. The places where many people mostly gathered include malls, parks and schools. When looking at the amount of kinetic energy produced by people in these places, or even on the dance floors of nightclubs, harvesting electricity from human activity makes sense. With this, the researcher thought of how to make something out of people's footsteps.

For this reason, the researcher aimed to convert the kinetic energy from a footstep into electricity and to use it as an alternative power supply for practical applications. At present, piezoelectric flooring is used to convert human footsteps to generate electricity. Piezoelectric effect generates an electric charge in response to applied mechanical stress (Umeda, et. al., 1997). Footsteps cause pressure on the floor, thus changing the shape of piezoelectric crystals. When stretched or deformed, these crystals produce an electric impulse and voltage is generated. The present research, on the other hand, incorporated springs instead of a piezoelectric material to 
generate electricity to reduce material costs. Moreover, piezoelectric materials can pick up stray voltages in connecting wires and the crystal is prone to cracking if overstressed. The crystals may also get affected by long use at high temperature (Animesh, 2015). For these reasons, the researcher developed and constructed an electricity generating device using a spring powered floor pad where a person's footstep will generate electricity. The electric generating floor mat will absorb the weight of people stepping on it in order to create electric energy. This can be accomplished by placing springs inside the floor mat. The converted electric energy will be stored in a battery which can be used to power electrical devices.

\section{OBJECTIVES OF THE STUDY}

This research aims to develop an electricity generating device using a spring powered floor pad. Specifically, the research sought to:

1. Design and construct an electricity generating device using a spring powered floor mat.

2. Determine the output voltage of the device for a particular number of steps.

3. Determine the discharging time of the battery for a particular number of steps.

\section{MATERIALS AND METHODS}

The researcher used the experimental method of research in this study. As pointed out by Key (1997), in this method, the researcher will maintain control over all factors that may affect the result of an experiment. In doing this, the researcher attempts to determine or predict what may occur. The research has four phases, the planning phase, design phase, assembling and the testing phase of the working prototype.

1. Planning Phase. At this phase, the researcher prepared and allocated the weeks for the different tasks involved.

2. Design Phase. In this phase, the researcher prepared the design of the prototype in CAD format and the circuit diagram of the generating system.

\subsection{Computer Aided Design Format of the Prototype's Design}

The researcher considered the use of available materials in the locality for ease of construction of the prototype device. Gear train pieces from electric fans and permanent magnet generator (PM) were incorporated in the prototype's design. Figure 1 shows the CAD model of the prototype device.

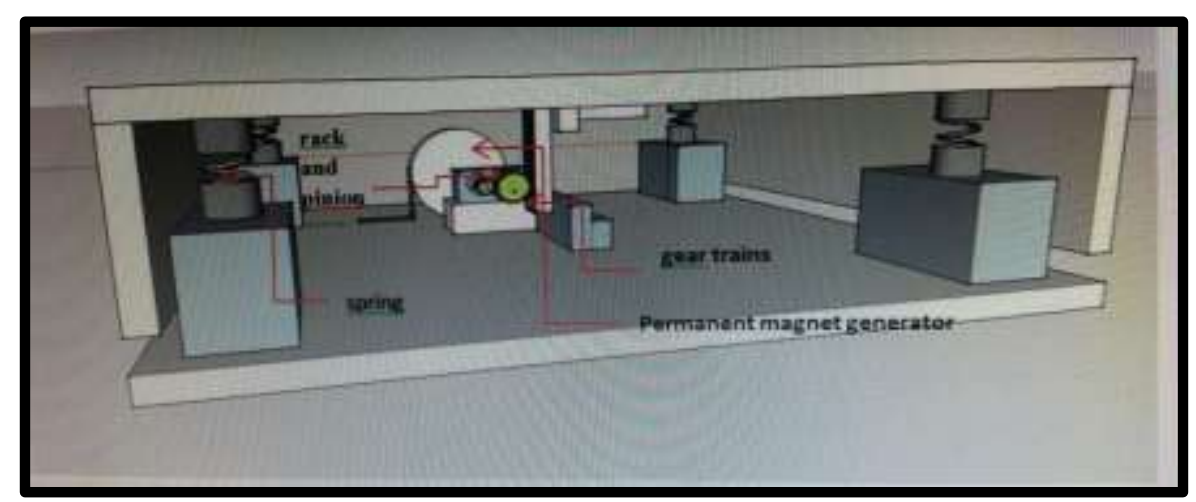

Figure 1 Computer Aided Design Model of the Prototype Device

The PM generator is a device that converts mechanical to electrical energy. The weight of the people stepping on the floor pad is the source of mechanical energy. The weight from their footsteps compresses the springs placed in the four corners of the floor mat. The rack and pinion mechanism is assembled below the floor pad. The pressure from the compression of the springs is transmitted to the linear gear bar called the rack which engages teeth on the circular gear called the pinion. The pinion is connected to the permanent magnet generator shaft. When the pinion rotates, the generator starts to rotate. Alternating current voltage is generated and is filtered to remove the alternating components. The LED lights connected to the generator lights up when electricity is generated. The energy produced is either stored in a battery or fed directly to electrical devices.

\subsection{Circuit Diagram of the Prototype Device}

The circuit diagram of the charging system is shown in Figure 2. The circuit is designed to convert the generated alternating current voltage to direct current by connecting a bridge rectifier circuit. A filter circuit removes the alternating current components of the rectified output and allows the direct current component to reach the load.

Building the complete system was developed by integrating the parts (i.e. rack and pinion, permanent magnet generator) in its casing with conformity to CAD of the prototype. The circuitry for charging the system was configured with regards to its circuit diagram. It was connected to the permanent magnet generator and was attached to the casing afterwards. 


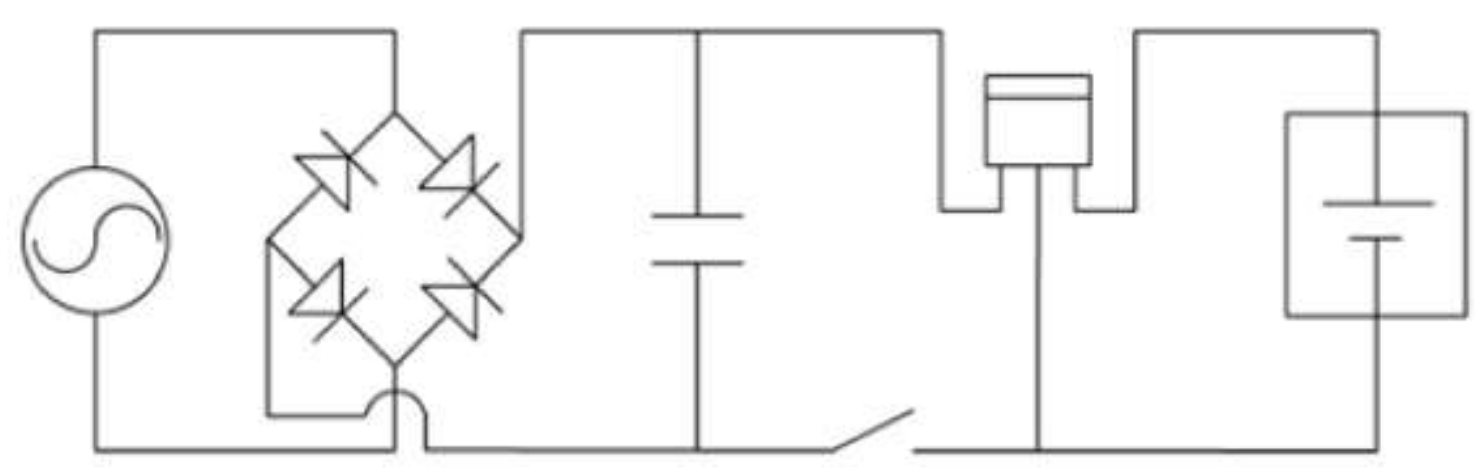

Figure 2. Circuit Diagram of the Charging System of the Electric Generating Floor Pad

3. Assembling Phase. This phase focused on building the prototype device in accordance to the Computer Aided Design. The following are the materials and equipment used in assembling the prototype of the device.

1. Permanent magnet generator

2. Charge controller circuit

3. Gear train (1 - 60 teeth, $1-14$ teeth)

4. Rack

5. Springs

6. Digital Multimeter

7. Plywood (for wooden casing)

8. Spray paint

9. Wood adhesives and other binding elements for the casing of the prototype device.

10. AA battery

4. Testing Phase. This phase involved the testing of the functions of the assembled prototype. A multimeter was connected in parallel with the permanent magnet (PM) generator to measure the voltage generated by the floor pad when it was stepped on. To determine the output voltage of the device with respect to the number of footsteps, the voltage is measured with each corresponding number of footsteps. Results indicate that the output voltage increases as the number of steps increases. An AA battery is connected to the generator to determine the discharging time of the battery for a particular number of steps. Results of the tests indicate that as the number of steps increases, the longer the discharging time of the battery.

\section{RESULTS AND DISCUSSIONS}

1. Design and Construction of An Electricity Generating Device Using A Spring Powered Floor Pad.

1.1 Design of the Electric Generating Floor Pad

The design of the electricity generating floor pad using springs works on the principle of conversion of linear motion into rotary motion due to application of footstep on the floor pad. The rotation of the generator produces electricity which can be stored in a battery or fed directly into small electrical devices. The researcher used four springs placed in the four corners of the floor pad as shown in Figure 1. When stepped on, the springs are compressed and the pressure from the compression is transmitted to the rack and pinion mechanism that drives the shaft of the permanent magnet generator. When the armature of the generator rotates, electricity is produced which lights up the LED lights connected to it. The interconnection of the different components of the floor pad is shown in Figure 3.

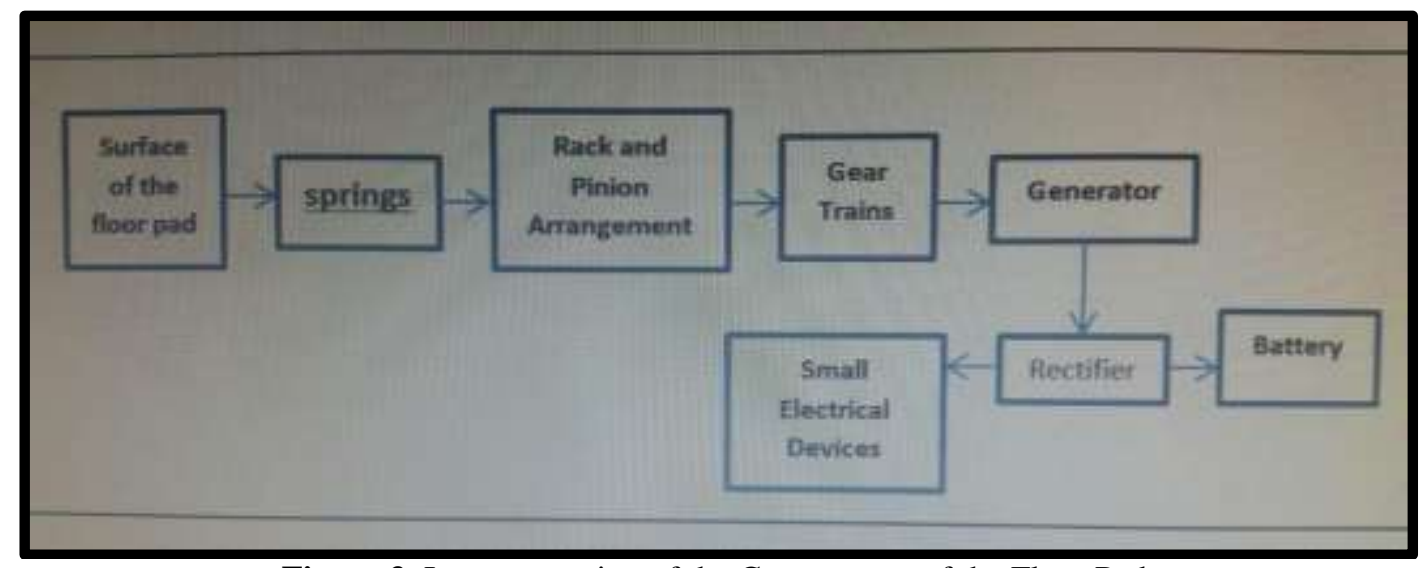

Figure 3. Interconnection of the Components of the Floor Pad 


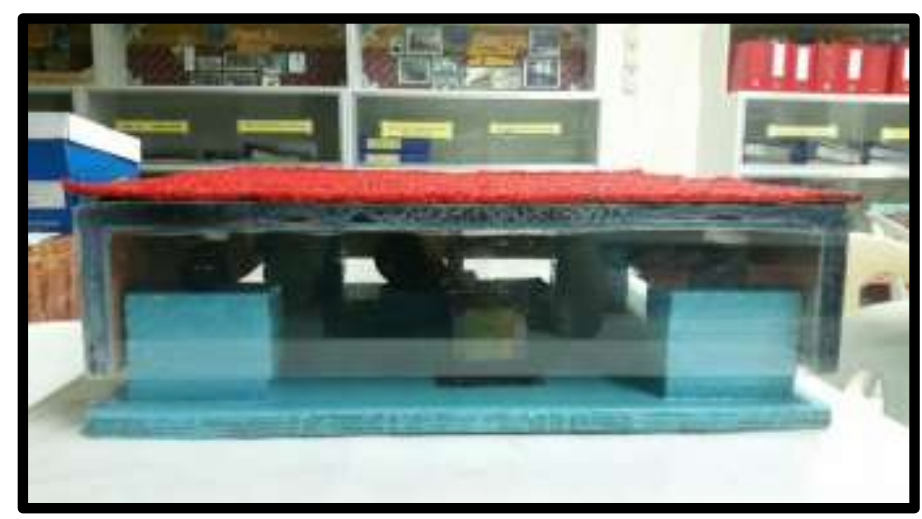

Figure 4. The Prototype Device

Figure 5 shows the main components of the prototype device. The main components of the device are the surface of the floor pad where people will step on; the springs located at the four corners beneath the surface of the floor pad that provide elastic potential energy upon compression; the rack and pinion which transmit the potential energy upon compression and release of the springs to the PM generator.
The PM generator converts this potential energy into electrical energy. The electrical energy is in the form of the voltage generated which is alternating current in nature. The bridge rectifier circuit in the circuit components of the prototype device removes the alternating nature of the voltage. The rectified direct current output is then connected to the LED lights.

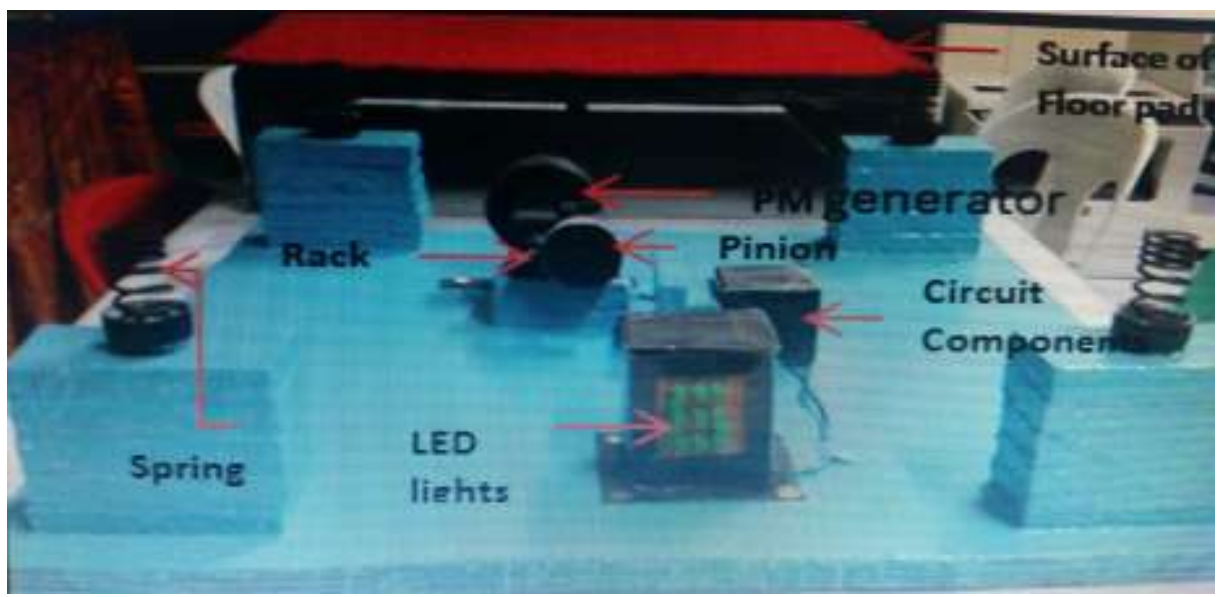

Figure 5. Components of the Prototype Device

\section{Performance of the Prototype Device}

The researcher assessed the device according to its performance during the testing. The results of the tests were considered in evaluating the functionality of the device.

\subsection{Number of Steps Vs Output Voltage of the Power Generating System}

Table 1 shows the number of steps with the corresponding output voltage. Results of the trials indicate that the output voltage increases as the number of steps increases. When a spring is stretched, the increased or decreased length is its extension. According to Hooke's Law, the extension of the spring is directly proportional to the force applied to it. More people stepping on the floor pad results in more force applied on the spring thereby creating a larger displacement. The potential energy of the spring increases as the displacement increases. This potential energy is then converted into electrical energy. Thus, more footsteps result in larger output voltage. In this research, output voltage has been regulated to $1.2 \mathrm{~V}$ using the constructed circuit supplying power to light the green LED lights.

Table 1. Number of Steps Vs. Output Voltage

\begin{tabular}{|c|c|c|c|}
\hline Trials & Number of Steps & Output Voltage & LED (Green) \\
\hline 1 & 1 & $3.00 \mathrm{~V}$ & Lights On \\
\hline 2 & 2 & $3.06 \mathrm{~V}$ & Lights On \\
\hline 3 & 3 & $3.12 \mathrm{~V}$ & Lights On \\
\hline 4 & 4 & $3.16 \mathrm{~V}$ & Lights On \\
\hline 5 & 5 & $3.20 \mathrm{~V}$ & Lights On \\
\hline
\end{tabular}




\subsection{Number of Steps Vs. Discharging Time of Battery}

The researcher conducted three trials to determine the discharging time of AA battery with respect to the number of steps. Table 2 shows the result of the experiment. The data indicate that as the number of steps increases, the longer the discharging time of the battery. Each footstep generates electricity which can be stored in a battery. With more footsteps, the stored energy in the battery accumulates so that the battery can be used for a longer period of time.

Table 2. Number of Steps Vs Battery Discharging Time

\begin{tabular}{|c|c|c|}
\hline Trials & Number of Steps & Discharging Time of AA Battery \\
\hline 1 & 100 & 30 seconds \\
\hline 2 & 200 & 40 seconds \\
\hline 3 & 300 & 45 seconds \\
\hline
\end{tabular}

\section{CONCLUSIONS}

The researcher has drawn the following conclusions from an extensive testing, observations and evaluation of the device.

1. The electric generating floor pad was designed with the use of springs in converting footsteps to electricity.

2. The electric generating floor pad generated AC output voltage that is rectified by a bridge circuit to direct current to power small electrical devices.

3. The output voltage increases as the number of steps increases and can be regulated and stored in a battery to power small electrical devices.

4. The battery has a longer discharging time with more footsteps applied to the floor pad.

\section{REFERENCES}

1. Animesh, Sacha, "Energy Generation By Using Piezoelectric Materials and Its Applications", //www.slideshare.net/AnimeshSachan/my-seminar4631388, March 26, 2015.

2. Key, James P., "Research Design in Occupational Education. Oklahoma State University", 1997.

3. Ripple, R.D., "Income and energy in the East Indian Ocean Basin. International Journal of Business Studies", 8, 1, June, 2000.

4. Umeda, M., Nakamura, K., and Ueha, S. Energy Storage Characteristics of a Piezogenerator Using Impact Vibration. Japan Journal of Applied Physics, Vol. 36, Part 1, No. 5b, pp.3146-3151, May 1997. 